\title{
Experimental and Discrete Dislocation Dynamics Approach to Initiation of Cleavage Fracture under Short Pulse Loads*
}

\author{
Huu Nhan TRAN $^{* *}$ and Hiroomi HOMMA ${ }^{* * *}$ \\ **Department of Mechanical Engineering, Toyohashi University of Technology \\ 1-1 Hibarigaoka, Tempaku-cho, Toyohashi 441-8580, Japan \\ E-mail: thnhanbkoto@homma-tut.jp \\ *** International Cooperation Center for Engineering \\ Education Development, Toyohashi University of Technology \\ 1-1 Hibarigaoka, Tempaku-cho, Toyohashi 441-8580, Japan
}

\begin{abstract}
Mode I crack initiation properties under stress intensity pulses with durations of 25, 50 and $100 \mu$ s are investigated by experiment and discrete dislocation dynamics. The cleavage fracture initiation is examined under the consideration of the pile-up of dislocations against an obstacle ahead of the crack tip and the coalescence of piled-up dislocations. In the experiment, under very short pulse loads with the duration of less than $50 \mu \mathrm{s}$, the dynamic fracture toughness, $K_{I d}$ remarkably increases and the crack tip opening displacement is closely correlated with $K_{I d}$. The numerical results are compared with the experimental ones in reasonable agreement.
\end{abstract}

Key words: Discrete Dislocation Dynamics, Cleavage Fracture, Short Pulse Load

\section{Introduction}

Cleavage fracture has played a crucial part in fracture problems, because it could lead to rapid crack propagation and eventually to catastrophic fracture of structures. Therefore, cleavage fracture has been intensively investigated. Many researchers ${ }^{(1)-(6)}$ discuss cleavage fracture from various aspects. Stroh ${ }^{(1),(2)}$ proposed formation of a cleavage crack by coalescence of planar edge dislocations piled up by a barrier. Smith ${ }^{(3)}$ examined theoretically cleavage crack initiation by pile up of dislocations on a single slip line. Oates ${ }^{(4)}$ experimentally investigated critical events for cleavage fracture. Their prudent observation indicated that at low temperature less than $-50^{\circ} \mathrm{C}$, the critical event for cleavage fracture in mild steel was nucleation of microscopic crack in carbide and at a temperature higher than $-50^{\circ} \mathrm{C}$, the critical event changed to grain-size crack propagation. Chen and Wang ${ }^{(5)}$ also observed the similar change in the critical event when the loading rate was increased. Recently, Kroon and Faleskog ${ }^{(6)}$ carried out numerical analysis on cleavage fracture initiation from a carbide crack to examine plastic rate-sensitivity, carbide shape, and stress triaxiality. In this analysis, finite element method was used to simulate cleavage crack propagation.

As mentioned above, cleavage fracture must be discussed with the critical event, crack nucleation or crack propagation. Noronha ${ }^{(7)-(9)}$ extensively investigated cleavage fracture from a microscopic crack existing on a crack line using a discrete dislocation dynamic model. In these studies, a two-dimensional coupling model in which a microscopic crack is located in the plastic zone ahead of a macroscopic crack is utilized. The fracture in this 
model is assumed to occur when the local stress intensity for the microscopic crack reaches a critical value. The applied stress intensity factor for the macroscopic crack then is defined as the fracture toughness.

Noronha and Farkas ${ }^{(10)}$ carried out an investigation into the effect of dislocation blocking distances on fracture behavior. In this investigation, the cleavage fracture stress, $\sigma_{f}$ was determined by the net tensile stress caused by the macroscopic crack fields and the array of dislocations emitted from the crack. The leading dislocation ceases moving when it reaches the obstacle located on the slip line.

In the previous work ${ }^{(11)}$, the authors can successfully represent the crack tip plasticity, especially crack tip opening displacement under short pulse loading by numerical analysis using a discrete dislocation dynamic model. This work aims to extend this model for crack tip plasticity to cleavage fracture initiation and obtain the insight into loading time effect on the cleavage fracture initiation from a crack subjected to very short pulse loading.

\section{The experiment}

The experimental apparatus for a one-point bend test consists of a gas gun, a projectile and a load transfer rod (see Ref.(12) for details). The pre-cracked specimen is laid without supports at the experimental apparatus contacting with the round end of the load transfer rod and an impact load is applied to the specimen through the load transfer rod to conduct one-point bend test. The applied load is gradually increased after each shot until the crack initiation is detected.

The low alloy carbon steel, JIS SM490A is used in the experiment. Three kinds of specimen configurations are prepared for the experiment. They have the same cross section of $40 \mathrm{~mm}$ height and $10 \mathrm{~mm}$ thickness, and three different lengths (spans) of 50, 100 and $180 \mathrm{~mm}$. A $17 \mathrm{~mm}$ long single-edged notch is machined in the center of each specimen to initiate a $3 \mathrm{~mm}$ long fatigue pre-crack by a hydraulic- servo fatigue machine.

Specimens are chilled at $-80^{\circ} \mathrm{C}$ and subjected to dynamic stress intensity pulses with different amplitudes. A dynamic stress intensity factor vs. time history is measured by the strain gage mounted near the crack tip. The method and the definition of the pulse duration in this work are the same as those used in the previous works ${ }^{(11)-(13)}$. The measured stress intensity could be approximated as a sinusoidal function of the time. The measured stress pulse durations are around 25, 50 and $100 \mu$ s for the smallest, middle and longest size of specimens, respectively. An example of the measured dynamic stress intensity is shown in Fig. 1 for three specimen geometries.

The crack growth is examined by optical microscope observation of the crack tip. The maximum value of the dynamic stress intensity pulse is plotted against the pulse duration with the legend of crack growth event in Fig. 2. The critical dynamic stress intensity for the crack initiation or dynamic fracture toughness, $K_{I d}$ is defined as the middle point between the minimum stress intensity value for crack growth and the maximum one for no crack growth.

Specimens subjected to higher stress intensity than $K_{I d}$ are fractured at the low temperature of $-200^{\circ} \mathrm{C}$. The stretched zone on the fractured surface near the initial crack tip can be clearly observed by SEM as shown in Fig. 3 .

Stretched zone width (SZW) is measured and the corresponding crack tip opening displacement (CTOD) is calculated according to JSME standard method. The average value of CTOD is defined as the critical crack tip opening displacement at fracture and is plotted as a function of stress intensity pulse duration in Fig. 4.

On the fractured surfaces observed by SEM, the nucleation origin of cleavage fracture is detected from the flow directions of river patterns by use of the procedure in Ref. 12 . Sharp edge of a chevron pattern, which is observed on a low magnified fracture surface, indicates a macroscopic fracture origin. Observing the flow directions of the river patterns 


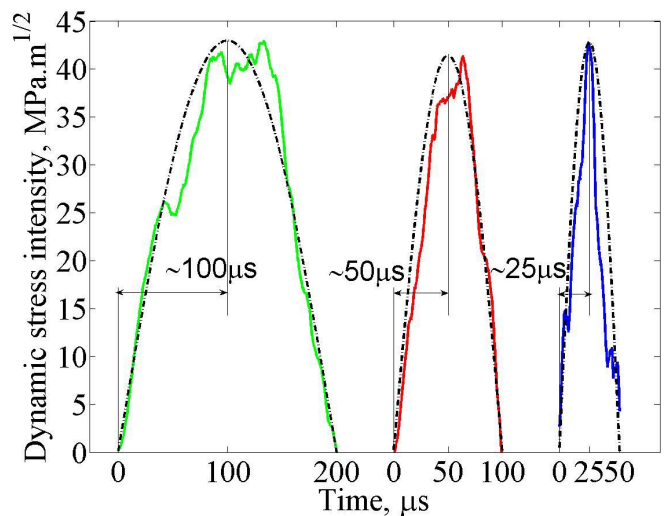

Fig. 1 Time history of dynamic stress intensity for three specimen geometries

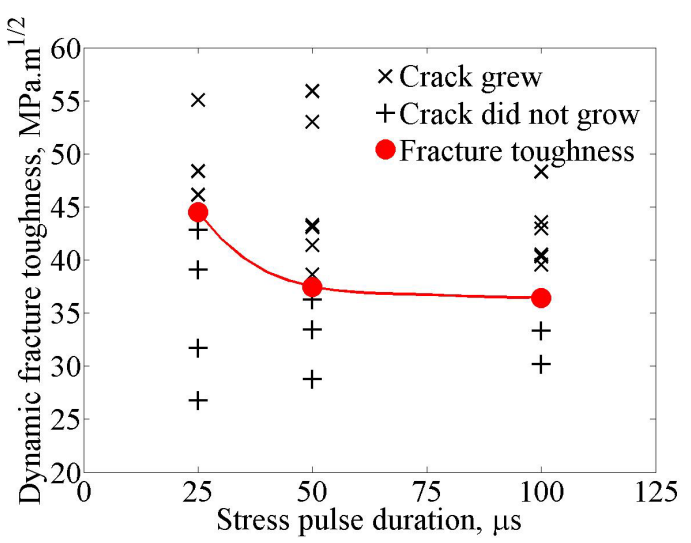

Fig. 2 Dynamic stress intensity as a function of stress pulse duration

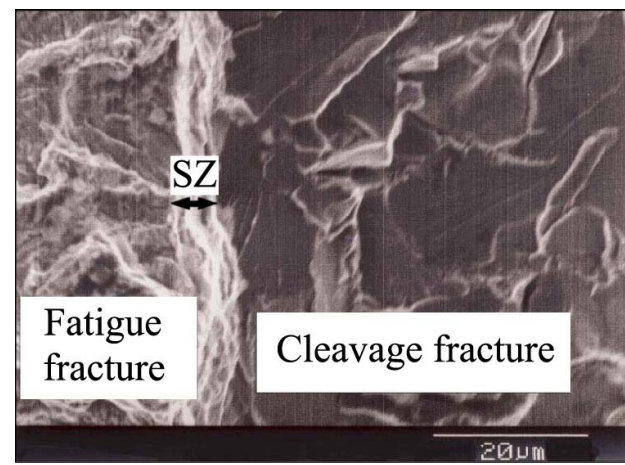

Fig. 3 The observed stretched zone

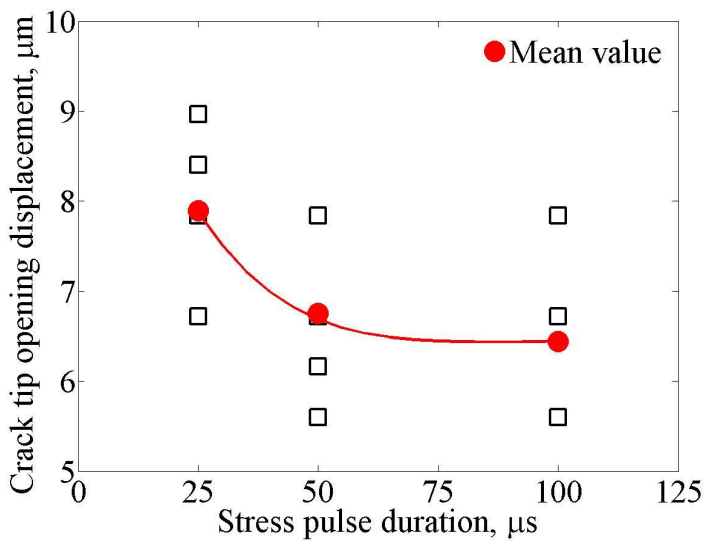

Fig. 4 The observed crack tip opening displacement 
with high magnification, the nucleation origin of the cleavage fracture can be detected, because all rivers are traced back to their flow origin. A cleavage fracture origin is located about $100 \mu \mathrm{m}$ ahead of the initial crack tip as shown by a head of the white arrow in Fig. 5.

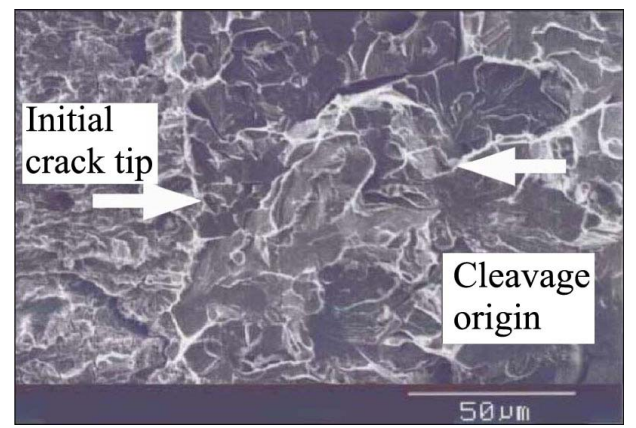

Fig. 5 Microscopic fractograph indicating cleavage fracture

The cleavage origin distance, $X_{c}$ is defined as the distance from the initial crack tip to the cleavage fracture origin and is measured on the photograph. The average value of $X_{c}$ for each stress pulse duration is measured and plotted as a function of stress intensity pulse duration in Fig. 6. It is seen that the cleavage origin distance is almost independent of the pulse duration.

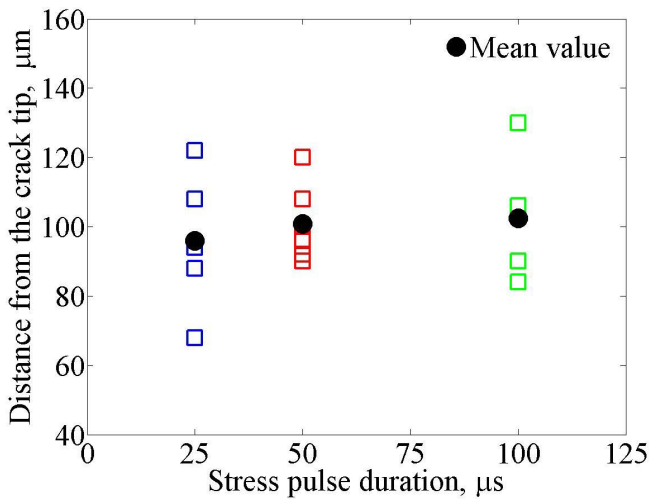

Fig. 6 Cleavage origin distance for three stress pulse durations

\section{Dislocation model and calculation parameters}

\subsection{Slip line model}

Because it is extremely complicated to deal analytically with real dislocation movement in a polycrystalline material such as SM490A used in the experiment, the previous work ${ }^{(11)}$ used a fairly simple model using discrete dislocation movement along a straight slip line in a homogeneous and isotropic material. Although the highly simplified model was used, the calculated result could agree well with the loading time effect on the crack tip opening displacement obtained by the experiment. Therefore, this work tries to extend the previous model to cleavage fracture initiation.

In the previous work ${ }^{(11)}$, a dislocation model is represented by four symmetrical arrays of discrete edge dislocations ahead of both crack tips. Two slip lines at each crack tip are inclined against the crack line at angles $\pm \alpha$ and $\pm(\pi-\alpha)$, respectively. A crack is embedded in an infinite isotropic elastic plane and is subjected to very short dynamic loading. Dislocations are emitted along four slip lines. The plastic zone is represented by a distribution range of discrete edge dislocations on the inclined slip line. In the current specimen configuration in this work, the crack length $2 a$ is $40 \mathrm{~mm}$. From simple calculation 
of stresses caused by a dislocation in an infinite plane, the order of the stress at the point 40 $\mathrm{mm}$ distant from a dislocation is around $E b /(10 a)$, where $E$ is Young's modulus, $b$ is Burgers vector and $0.25 \mathrm{~nm}$ indicated later. It is roughly $E \times 10^{-9}$. Therefore, the effect of the dislocations on two slip lines at the other crack tip could be neglected to calculate stresses at dislocation positions on the slip lines at a considered crack tip.

Instead of four arrays of discrete edge dislocations in the previous work ${ }^{(11)}$, the present dislocation model is represented by only two arrays at a crack tip in the plane strain condition. The slip lines are symmetric and inclined against the crack plane at angles $\pm \alpha$ in an infinite elastic plane. The edge dislocations will be emitted on the slip lines. Hence, the Burgers vectors are parallel to the slip lines and the unit vector components are $(\cos \alpha$, $\sin \alpha),(-\cos \alpha, \sin \alpha)$ for the inclined slip lines of the number 1 and 2 , respectively. The crack leis along the $\mathrm{x}$ axis, and its length is $40 \mathrm{~mm}$, which is subjected to short impulsive loading under a mode I condition. The schematic illustration of the dislocation model is shown in Fig.7, but only the two continuous dark-colored slip lines are considered.

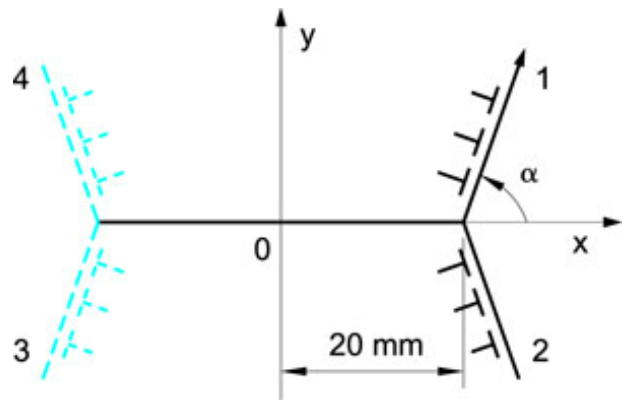

Fig. 7 Schematic illustration of the dislocation model

The elastic stress components are calculated by complex potential functions for the plane strain condition. Two complex potential functions $\phi(z)$ and $\psi(z)$ are used as follows:

$$
\sigma_{x x}+\sigma_{y y}=2\left(\phi^{\prime}(z)+\overline{\phi^{\prime}(z)}\right), \quad \sigma_{y y}-\sigma_{x x}+2 i \tau_{x y}=2\left(\bar{z} \phi^{\prime \prime}(z)+\psi^{\prime}(z)\right)
$$

where $z=x+i y$ is a complex variable, the bar over a variable denotes complex conjugate, $\bar{z}=x-i y$, and $i=(-1)^{1 / 2}$, the prime (') and (") denote the first and the second derivative of the complex potential functions.

A dislocation at a position on the slip line ahead of a crack tip raises stresses surrounding it. Because the dislocation exists near a crack, the traction stress and shear stress must be zero on the crack surface. V. Vitek et al. ${ }^{(14)}$ composed the complex stress potential functions from stress functions for a dislocation in an infinite plane and stress functions to eliminate the traction stress and the shear stress on the crack surface raised by the former stress function. The image stress is defined as the stress calculated by the later stress function. In this work, their stress functions are used to calculate the stresses raised by a dislocation.

To determine the stress field of the crack subjected to a uniform applied stress $\sigma^{a}$, the complex stress functions are given as:

$$
\phi^{c+a}(\zeta)=\frac{1}{8} \sigma^{a} a(\zeta-3 / \zeta), \quad \psi^{c+a}(\zeta)=\frac{1}{4} \sigma^{a} a\left[\zeta+1 / \zeta-4 \zeta /\left(\zeta^{2}-1\right)\right]
$$

where $a$ is the half length of crack, and $z=\frac{1}{2} a(\zeta+1 / \zeta)$.

The stress field of an edge dislocation with Burgers vector $\left(b_{x}, b_{y}\right)$ located at $\left(x_{d}, y_{d}\right)$ in a smooth infinite plane is determined by:

$$
\phi^{d}(z)=\gamma \ln \left(z-z_{d}\right), \quad \quad \psi^{d}(z)=\bar{\gamma} \ln \left(z-z_{d}\right)-\gamma \overline{z_{d}} /\left(z-z_{d}\right)
$$


where $z_{d}=\frac{1}{2} a\left(\zeta_{d}+1 / \zeta_{d}\right)$ and $\gamma=[G / 4 \pi(1-v)]\left(b_{y}-i b_{x}\right), G$ is shear modulus, and $v$ is Poisson's ratio

In case of a cracked infinite plane, the image stress field can be determined by the complex stress functions as:

$$
\begin{aligned}
\phi^{i m}(\zeta)= & 2 \gamma \ln \zeta-\gamma \ln \left(\zeta-1 / \zeta_{d}\right)-\gamma \ln \left(\zeta-1 / \overline{\zeta_{d}}\right)+ \\
& +\bar{\gamma}\left[\zeta_{d} \overline{\zeta_{d}}\left(\overline{\zeta_{d}^{2}}-1\right)\right]^{-1}\left[\zeta_{d}\left(1+\overline{\zeta_{d}^{2}}\right)-\overline{\zeta_{d}}\left(\zeta_{d}^{2}+1\right)\right]\left[\zeta-1 / \overline{\zeta_{d}}\right]^{-1}, \\
\psi^{i m}(\zeta)= & 2 \bar{\gamma} \ln \zeta-\bar{\gamma} \ln \left(\zeta-1 / \zeta_{d}\right)-\bar{\gamma} \ln \left(\zeta-1 / \overline{\zeta_{d}}\right)+ \\
& +\gamma\left[\zeta_{d} \overline{\zeta_{d}}\left(\zeta_{d}^{2}-1\right)\right]^{-1}\left[\overline{\zeta_{d}}\left(1+\zeta_{d}^{2}\right)-\zeta_{d}\left(\overline{\zeta_{d}^{2}}+1\right)\right]\left[\zeta-1 / \zeta_{d}\right]^{-1}+ \\
& -\zeta\left[\left(1+\zeta^{2}\right) /\left(\zeta^{2}-1\right)\right] \phi^{i m^{\prime}}(\zeta)
\end{aligned}
$$

The stress components for an edge dislocation on the inclined slip line ahead of crack tip subjected to a uniform applied stress can be determined by summation of each stress component calculated from the equations (1) to (4). Then, the shear stress acting on an inclined slip line rotated from the crack line by an angle $\alpha$ can be found as:

$$
\tau=\frac{\sigma_{y y}-\sigma_{x x}}{2} \sin 2 \alpha+\tau_{x y} \cos 2 \alpha
$$

After $n$ dislocations have been emitted, the resolved shear stress applied to the $i^{\text {th }}$ dislocation located at $z_{i 1}$ on an inclined slip line number 1 , is obtained as:

$$
\tau^{r e s}\left(z_{i 1}\right)=\tau^{c+a}\left(\sigma^{a}, z_{i 1}\right)+\tau^{i m}\left(z_{i 1}, z_{i 1}\right)+\sum_{j=1}^{2}\left\{\sum_{k=1(j=1,1, k \neq i)}^{n}\left[\tau_{j}^{d}\left(z_{i 1}, z_{k j}\right)+\tau_{j}^{i m}\left(z_{i 1}, z_{k j}\right)\right]\right\}
$$

where $z_{k j}$ is the position of the $k^{t h}$ dislocation on the inclined slip line of the number $j$, and $i$ and $j$ denote the dislocation number and the slip line number, respectively. The equation consists of three parts: the shear stress caused by the crack subjected to the uniform applied stress $\sigma^{a}$, the image shear stress caused by the dislocation at $z_{i 1}$, and the summation of direct interaction shear stresses induced by all the other dislocations and their image stresses.

\subsection{Dislocation emission and movement from the crack tip}

For the symmetry, the same phenomena happen on two slip lines. Dislocation emission source is placed on the slip line and at the position $z_{0 j}$ close to the crack tip. At each time step, the resolved shear stress at the source position, $\tau^{r e s}\left(z_{0 j}\right)$ is computed. A dislocation is emitted from the source when $\tau^{\text {res }}\left(z_{01}\right)$ exceeds a critical value. The friction shear stress, $\tau_{f}$, the material resistance against the dislocation movement, is used for the critical value in the same consideration as the other researchers ${ }^{(7),(8),(10)}$. When the dislocation emission criterion is satisfied, a new dislocation will be emitted and allowed to move away from the source. Furthermore, the resolved shear stresses at all the emitted dislocation positions are computed and compared with $\tau_{f}$ to determine the dislocation movement. Dislocations subjected to the resolved shear stresses larger than $\tau_{f}$ will move at a certain traveling velocity along the inclined slip line.

The empirical dislocation traveling velocity law ${ }^{(7),(8)}$, which is related to the resolved shear stress at each dislocation position, is used in this work: 


$$
\left.\begin{array}{l}
\left|\tau^{\text {res }}\left(z_{i}\right)\right|<\tau_{f} \Rightarrow v=0, \\
\left|\tau^{\text {res }}\left(z_{i}\right)\right|>\tau_{f} \Rightarrow v=A\left(\frac{\left|\tau^{\text {res }}\left(z_{i}\right)\right|-\tau_{f}}{\tau^{\text {res }}\left(z_{i}\right)}\right)\left|\tau^{\text {res }}\left(z_{i}\right)\right|^{m}
\end{array}\right\}
$$

The first equation shows that if the resolved shear stress $t^{\text {res }}\left(z_{i j}\right)$ is less than the critical stress, the dislocation does not move. The second equation shows that if the resolved shear stress is greater than the critical stress, the dislocation will move at the velocity proportional to excess extend from the critical value and the $m^{-t h}$ power of the resolved shear stress. The appropriate values of $A$ and $m$ have been chosen to achieve a reasonable agreement between the numerical and experimental ones.

Because the applied load is a function of the time, above all equations are calculated in the time domain. To obtain converged and consistent results, the applied load-time history is divided into very short time intervals and in those time intervals, it is assumed that the applied load is constant, and all the dislocations travel at constant velocities. At the beginning of every time interval, resolved shear stresses are calculated and then the traveling velocities of all the emitted dislocations are calculated. Then, traveling distances of all the dislocations are evaluated to determine new positions of the dislocations at the end of every time interval. The total number of emitted and traveling dislocations gradually increases from the first stage with passage of the time.

\subsection{Cleavage fracture model}

In this work, it is hypothesized that initiation of the cleavage fracture results from pile-up of dislocations at an obstacle located on the slip line such as a grain boundary, an inclusion, and a carbide lathe like Smith's model ${ }^{(3)}$. The obstacle is modeled as an impenetrable barrier at which dislocations will pile-up. Thus, the leading dislocation ceases moving near the obstacle and pile-up of dislocations is formed as illustrated in Fig.8.

The pile-up of the dislocations raises stress concentration near the leading dislocation. Coalescence of the piled-up dislocations could happen when two dislocations approach each other within a critical distance. It might be reasonable to define the critical distance as the magnitude of Burgers vector. Coalescence of the dislocations happens between the leading dislocation and the neighbor one. Then, the Burgers vector of the leading dislocation increases in the magnitude by that of neighbor dislocation Burgers vector.

For this dislocation pile-up model, it is hypothesized that the cleavage fracture initiation takes place when the principal stress in front of the obstacle exceeds the cleavage stress of this material.

\subsection{Model parameters}

(1) Material property

Young's modulus and Poisson ratio: The material for the numerical analysis should be the same as one used in the experiment. Then, Young's modulus, $E$ is $206 \mathrm{GPa}$ and the Poisson ratio $v$ is 0.3 .

Dynamic yield strength, $\sigma_{y d}$ is considered dependent on stress pulse duration and temperature. Stress pulse duration effect on $\sigma_{y d}$ could be approximately estimated by the experimental results on the crack tip opening displacement ${ }^{(11)}$ and the formula of crack tip opening displacement ${ }^{(15)}$,

$$
\delta=0.6 K_{I}^{d y n^{2}} /\left(E \sigma_{y d}\right)
$$

The experimental results of $\delta$ were $9.9,10.9$ and $12.1 \mu \mathrm{m}$ for 25,50 and $100 \mu$ stress intensity pulse durations, respectively ${ }^{(11)}$, under the applied dynamic stress intensity of $60 \mathrm{MPa} \cdot \mathrm{m}^{1 / 2}$ at room temperature. Then, the temperature dependence of $\sigma_{y d}$ is interpolated by the obtained experimental data ${ }^{(16)}$. At the present experimental condition, 
the dynamic yield strength is estimated as 1187,1083 and $988 \mathrm{MPa}$ for 25,50 and 100 $\mu$ s stress pulse durations respectively, at $-80^{\circ} \mathrm{C}$.

The cleavage strength of the material is determined as $4.2 \mathrm{GPa}$ so that the experimental data on dynamic fracture toughness could be consistent with the model proposed here.

(2) Slip line and dislocation property

The material parameters $A$ and $m$ in the dislocation velocity law, Eq. 7 are taken as $4 \times 10^{-3}$ and 1.75 , respectively.

The friction shear stress $\tau_{f}$, which is the material resistance against the dislocation movement, can be taken arbitrarily, but it is considered closely related to the yield strength. Hence, $\tau_{f}$ is taken as $\sigma_{y d} / 5$.

A dislocation emission source on the slip line is placed at the position $200 \mathrm{~b}, 50 \mathrm{~nm}$ distant from the crack tip. This point is determined as the nearest one that can provide stable numerical results.

The angle $\alpha$ between the slip line and the crack line is taken as $70.5^{\circ}$, which is the direction of the maximum shear stress in the elastic crack tip field.

The magnitude of Burgers vector is taken as $b=0.25 \mathrm{~nm}$, which is common to discrete dislocation dynamics simulations.

The cleavage fracture origin measured on the photograph taken by a scanning electronic microscope (SEM) in the experiment, $X_{c}$ is around $100 \mu \mathrm{m}$ as shown in Fig.6. This is the projected length onto the crack line. Therefore, in this model, the obstacle as a cleavage fracture origin is placed at the position, $300 \mu \mathrm{m}\left(\mathrm{X}_{\mathrm{c}} / \cos \alpha\right)$ distant from the crack tip on the inclined slip line. The size of the obstacle is taken as $10 \mu \mathrm{m}$, which is nearly equal to the average size of cleavage facet at cleavage origin. Thus, the local tensile stress, $\sigma_{y y}$ at the site of the cleavage origin is computed.

\subsection{Calculation parameters}

Time interval $\Delta t$ is taken so small as $1 \times 10^{-2}$ ns to prevent a dislocation from overshooting the others at each time step or to make sure that numerical stability and convergence is guaranteed.

\section{Results and discussion}

For each step, the resolved shear stresses of all the emitted dislocations are computed. Therefore, the computing time is significantly affected by the number of dislocations. To reduce the computing time, the bundled-dislocation technique, proposed by Roberts ${ }^{(17)}$, was utilized. A super dislocation of rank 2 is generated by bundling three dislocations of rank 1 after the ninth dislocation has been emitted. This process is repeated whenever the ninth dislocation of rank $n \in N$ is created.

The specimen geometry and loading configuration are apparently different in the experiment and the discrete dislocation dynamics model as elaborated in the previous sections. However, connection between the model and the experimental conditions in section 2 can be made as follow. Because stress field ahead of a crack tip is uniquely established by a stress intensity factor if the considered region is much smaller than the crack length, the similar stress intensity to one in the experiment is applied to the crack in the model.

To examine cleavage fracture initiation, the local tensile stress $\sigma_{y y}$ at the cleavage origin $z_{c 1}$ on the inclined slip line number 1 , shown in Fig. 8 is calculated by the same concept as the calculation of the resolved shear stress as:

$$
\sigma_{y y}=\sigma_{y y}^{c+a}\left(\sigma^{a}, z_{c 1}\right)+\sum_{j=1}^{2} \sum_{i=1}^{n}\left\{\sigma_{y y j}^{d}\left(z_{c 1}, z_{i j}\right)+\sigma_{y y j}^{i m}\left(z_{c 1}, z_{i j}\right)\right\}
$$




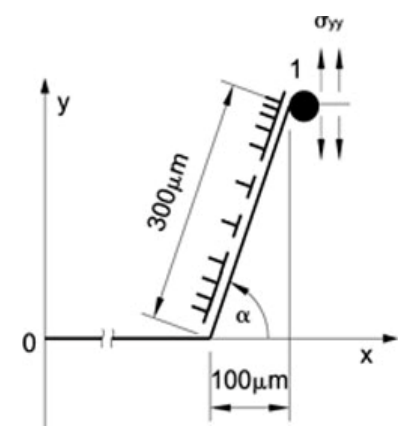

Fig. 8 Local tensile stress illustrated schematically

At first, dislocation distributions on the inclined slip line are calculated for three durations of stress pulse in the experiment. The applied uniform stress $\sigma^{a}$ used in the calculation is the quasi-static uniform applied stress sustained by a $40 \mathrm{~mm}$ long crack in an infinite plane and causes the quasi-static stress intensity corresponding to the dynamic fracture toughness $K_{I d}$ obtained in the experiment for $100 \mu$ s stress pulse duration. Then, the values of $\sigma_{y y}$ at the cleavage fracture origin are calculated for three pulse durations. The calculated results are shown as a function of time in Fig. 9.

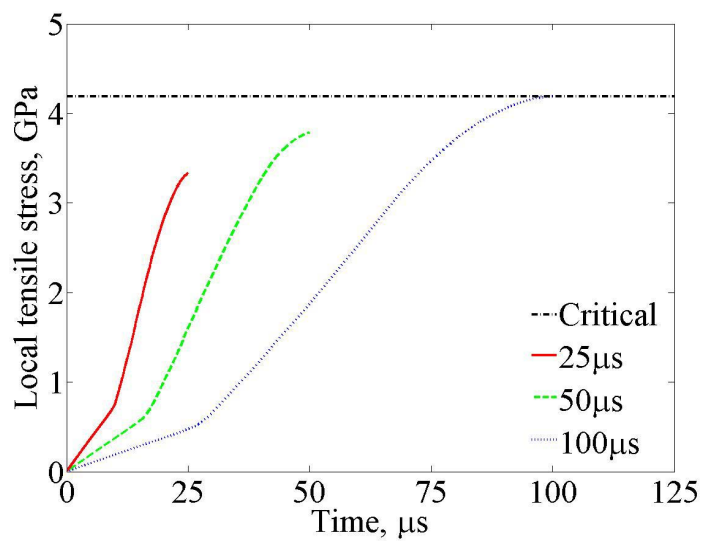

Fig. 9 Local tensile stress as a function of time

At the initial stage of pulse duration, $\sigma_{y y}$ gradually increases only by increment in the applied load. Then, $\sigma_{y y}$ steeply increases with the pile-up of dislocations after the leading dislocation reaches the position of the obstacle. The shorter pulse duration is, the smaller value of $\sigma_{y y}$ is. The obtained values of $\sigma_{y y}$ for $25 \mu$ s and $50 \mu$ s pulse durations are as $79.5 \%$ and $90.3 \%$ small as the one for $100 \mu \mathrm{s}$, respectively. This could be explained by the fact that for $25 \mu \mathrm{s}$ and $50 \mu$ s pulse durations, the loading time is so short that the emitted dislocations from the crack tip could not have time to arrive at positions close to the obstacle and to contribute in enhancement of the stress at the obstacle.

The cleavage fracture stress is postulated as the calculated $\sigma_{y y}, 4.2 \mathrm{GPa}$ for $100 \mu$ s pulse duration. Then, a certain value of the applied stress $\sigma^{a}$ is taken for trial for the other pulse durations. The $\sigma_{y y}$ is calculated for the trial $\sigma^{a}$, and if the $\sigma_{y y}$ is smaller than the cleavage fracture stress postulated above, the trial $\sigma^{a}$ is increased until the $\sigma_{y y}$ exceeds the cleavage fracture stress.

Fracture toughness values are calculated by the obtained critical $\sigma^{a}$ for three pulse durations. The calculated fracture toughness is compared with the experimental results in Fig. 10. It should be noted that the fracture toughness calculated by the proposed model can well predict the experimental results. 


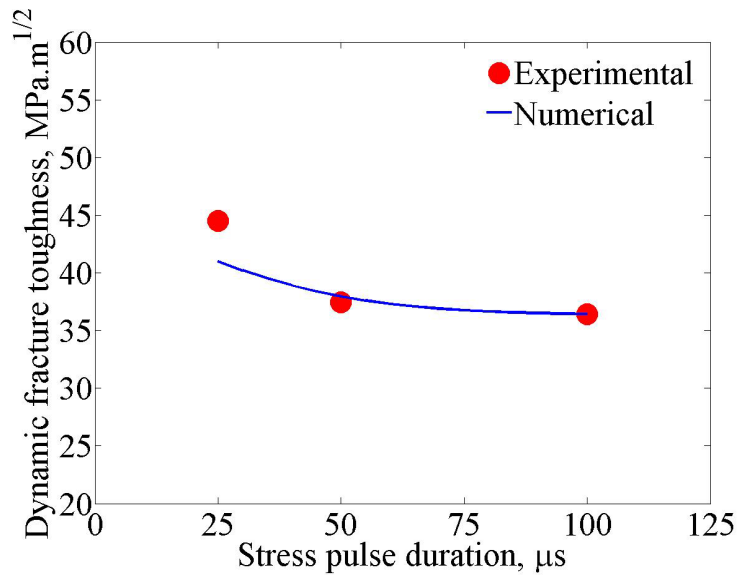

Fig. 10 Fracture toughness

Dislocation density distribution along the slip line is estimated for three stress pulse durations. Dislocation density is defined as a line density $0.5\left(b_{i}+b_{i-1}\right) /\left(r_{i}-r_{i-1}\right)$, where $r_{i}$ is the distance from the crack tip to the $i^{\text {th }}$ bundled dislocation and $b_{i}$ is the magnitude of Burgers vector. The density distribution on the slip line is plotted as a function of the distance from the crack tip for three pulse durations in Fig. 11. It is seen in Fig. 11 that under the shorter pulse duration, the dislocation density distribution is slightly higher than the longer one.

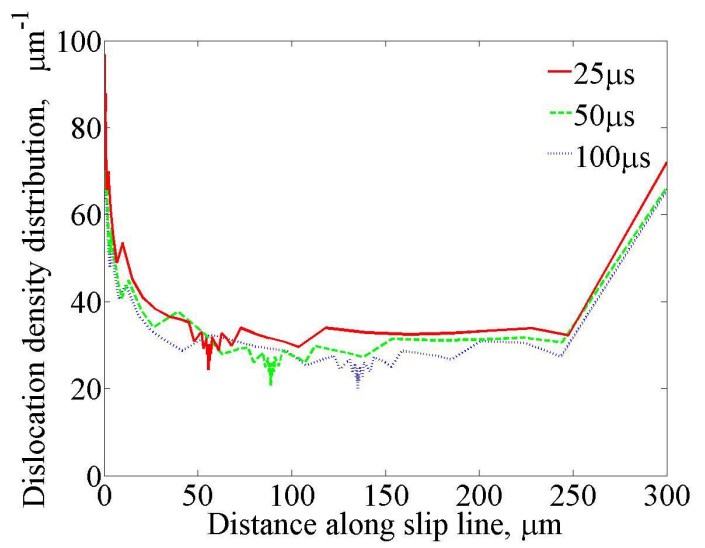

Fig. 11 Dislocation density distribution along the slip line for three pulse durations

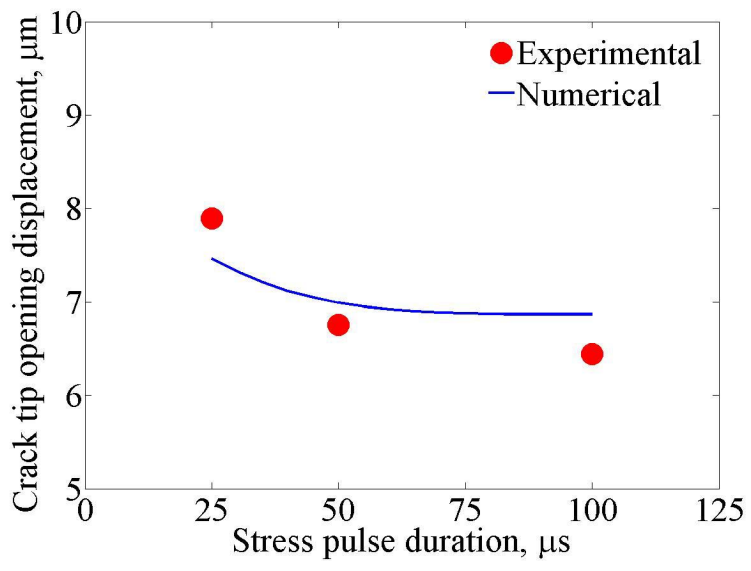

Fig. 12 Crack tip opening displacement 
The crack tip opening displacement (CTOD) can be also calculated by:

$$
C T O D=2 n_{d} b \sin \alpha
$$

where $n_{d}$ is the number of emitted dislocations. The numerical results are also well compared with the measured results as shown in Fig. 12.

\section{Conclusions}

Dynamic cleavage fracture of the low alloy carbon steel, JIS SM490A was carried out by one-point bend test at low temperature of $-80^{\circ} \mathrm{C}$. The numerical simulation to the experiment was carried out by the discrete dislocation dynamics model of cleavage fracture considering the pile-up of dislocations and coalescence of piled-up dislocations. Then, the following conclusions are deduced:

- The remarkable increase in the dynamic fracture toughness value, $K_{I d}$ was obtained under high loading rate or short pulse duration.

- The value of CTOD was also closely correlated with $K_{I d}$.

- The cleavage origin distance is almost independent of the pulse duration and equal to around $100 \mu \mathrm{m}$.

- The numerical results of $K_{I d}$ and CTOD under three pulse durations were compared with the experimental results. The good agreement between the both results suggested that the used discrete dislocation dynamics model could be reasonable and valid.

\section{References}

(1) Stroh, A.N., The formation of cracks as a result of plastic flow, Proceedings of the Royal Society of London, Series A, Mathematical and Physical Sciences, Vol. 223 (1954), pp. 404-414.

(2) Stroh, A.N., The formation of cracks in plastic flow II, Proceedings of the Royal Society of London, Series A, Mathematical and Physical Sciences, Vol. 232 (1955), pp. 548-560.

(3) Smith, E., Cleavage fracture in mild steel, International Journal of Fracture, Vol. 4, No. 2, (1968), pp. 131-145.

(4) Oates, Effect of temperature and strain rate on cleavage fracture in a mild steel and a low-carbon manganese steel, J Iron Steel Inst (1969), pp. 353-357.

(5) Chen, J. H. and Wang, G. Z., Change of critical event for cleavage fracture of HSLA steel, Proceedings of ICF11, Italy, 27, Metallic Materials, 2898 (2005).

(6) Kroon, M. and Faleskog, J., Micromechanics of cleavage fracture initiation in ferrite steels by carbide cracking, J. of Mechanics and Physics of Solids, Vol. 53, (2005), pp. 171-196.

(7) Roberts, S.G., Noronha, S.N., Wilkinso, A.J. and Hirsch, P.B., Modelling the initiation of cleavage fracture of ferritic steels, Acta Mater., Vol. 50 (2002), pp. 1229-1244.

(8) Noronha, S.J., Huang, J., Ghoniem, N.M., Multiscale Modeling of the Brittle to Ductile Transition, J. Nucl. Mater., 3292333 (2004), pp. 1180-1184.

(9) Noronha, S.J., Ghoniem, N.M., Dislocation Simulation of Brittle to Ductile Transition in Ferritic Steels, Metallurgical and Materials Transaction A, Vol. 37A (2006), pp. 539-544.

(10) Noronha, S.J., Farkas, D., Effect of dislocation blocking on fracture behavior of $\mathrm{Al}$ and $\alpha$-Fe: a multiscale study, Materials Science and Engineering A365 (2004), pp. 156-165.

(11) Homma, H., Tran, H.N., Crack tip plasticity by classic dislocation dynamics, Advanced 
Materials Research, Vols. 33237 (2008), pp. 97-102.

(12) Homma, H., Kanto, Y., Tanaka, K., Cleavage fracture under short stress pulse loading at low temperature, ASTM STP 1130. Philadelphia: American Society for Testing and Materials, 1992, pp. 37-49.

(13) Rizal, S., Homma, H., Nazer, M. and Kishida, E., Experimental approach to dimple fracture mechanisms under short pulse loading, Engineering, Fracture Mechanics, Vol. 69, No. 12 (2002), pp. 1377-1390.

(14) Vitek, V., Yielding on inclined planes at the tip of a crack loaded in uniform tension, Mech. Phys. Solids, Vol. 24, No. 5 (1976), pp. 263-275.

(15) Chen, J.H., Wang, G.Z., Study on cleavage fracture criteria of the quasi-brittle and micro-inhomogeneous materials, International Journal of Fracture 108 (2001): pp. 143-164.

(16) Chen, J.H., Wang, Q., Wang, G.Z., Li, Z., Fracture behavior at crack tip - a new framework for cleavage mechanism of steel, Acta Materialia 51 (2003), pp. 1841-1855

(17) Roberts, S.G., Modelling the brittle to ductile transition in single crystals, Computer Simulations in Materials Science (Series E: Applied Sciences), Vol. 308 (1996), pp. 409-433. 